\title{
Silk Fibroin/Nano-CaP Bilayered Scaffolds for Osteochondral Tissue Engineering
}

\author{
Le-Ping Yan ${ }^{1,2, a}$, Joaquim M. Oliveira, ${ }^{1,2, b}$, Ana L. Oliveira ${ }^{1,2,3, c}$ \\ and Rui L. Reis ${ }^{1,2, d}$ \\ ${ }^{1} 3 \mathrm{~B}$ 's Research Group - Biomaterials, Biodegradables and Biomimetics, University of Minho, \\ Headquarters of the European Institute of Excellence on Tissue Engineering and Regenerative \\ Medicine, AvePark, S. Cláudio de Barco, 4806-909 Taipas, Guimarães, Portugal. \\ ${ }^{2}$ ICVS/3B's - PT Government Associate Laboratory, Braga/Guimarães, Portugal. \\ ${ }^{3}$ Department of Health Sciences, Portuguese Catholic University, 3504-505 Viseu, Portugal. \\ ayan.leping@dep.uminho.pt, ${ }^{b}$ miguel.oliveira@dep.uminho.pt, canaleite@dep.uminho.pt, \\ 'rgreis@dep.uminho.pt (corresponding author)
}

Keywords: Osteochondral Tissue Engineering; Bilayered Scaffold; Silk Fibroin; Nano Calcium Phosphate.

\begin{abstract}
In this study, bilayered silk and silk/nano-CaP scaffolds were developed for osteochondral (OC) tissue engineering. Aqueous silk solution (16 wt.\%) was used for preparation of the cartilage-like layer and, for generation of the silk/nano-CaP suspension and the bottom layer (CaP/Silk: 16 wt.\%). The scaffolds were formed by using salt-leaching/lyophilization approach. The scanning electron microscopy revealed that the both layers presented porous structure and integrated well. Micro-computed tomography images confirmed that the $\mathrm{CaP}$ phase was only retained in the silk/nano-CaP layer. The hydration degree and mechanical properties of the bilayered scaffold were comparable to the ones of each single layer. The apatite crystal formation was limited to the silk/nano-CaP layer, when soaking the scaffold in a simulated body fluid solution, which is a must for the application of the developed scaffolds in OC tissue engineering.
\end{abstract}

\section{Introduction}

Articular cartilage is located in the joint and plays an important role for absorbing the shock and facilitating the joint mobility with extreme low friction [1]. Articular cartilage defects are usually irretrievable as cartilage is an avascular tissue and lack of access to potential reparative factors [1]. As the cartilage defect progresses, it would reach subchondral bone and form osteochondral defect [2]. Osteochondral defects (OCD) involves injuries in the articular cartilage and subchondral bone which are integrated but of distinct biomechanical properties tissues. Proper surgical treatments should be performed to prevent the OCD develop toward chronic disease [3]. Currently, joint debridement, microfracture, and mosaicplasty are used for the treatment of OCD less than $2.5 \mathrm{~cm}^{2}$ [3]. For OCD larger than $2.5 \mathrm{~cm}^{2}$, autologous chondrocyte implantation (ACI) is employed [3]. All of the above mentioned clinical approaches can only partially or transiently repair the OCD, but the ideal long-term regeneration is not achieved yet [3]. Tissue engineering has been emerging as a promising alternative approach for the regeneration of OCD [4]. Taking into account the properties of the connected articular cartilage and subchondral bone tissues, increasing attentions have been addressed in the development of bilayered scaffolds for OCD regeneration [2,3]. Our group has been working in OCD regeneration strategies over the last years [5]. Previously, we have worked on the development of high strength silk and silk/nano-CaP based scaffolds [6,7]. In the present study novel bilayered silk and silk/nano-CaP scaffolds were produced and characterized for finding application in OCD regeneration.

\section{Materials and Methods}

Silk fibroin was extracted from Bombyx Mori cocoons (Portuguese Association of Parents and Friends of Mentally Disabled Citizens). Concentrated aqueous silk fibroin solutions (16 wt.\%) were prepared as previously reported [6]. For the creation of bilayered scaffolds, silk/nano-CaP 
composite was first produced. Silk/CaP suspension was developed via an in-situ synthesis method, namely by addition of equal volume calcium chloride solution (6 mol/L, Sigma) and ammonia dibasic phosphate solution ( $3.6 \mathrm{~mol} / \mathrm{L}$, Sigma) into the aqueous silk solution. The $\mathrm{pH}$ of the formed suspension was adjusted to 8.5 by using ammonia (30\%, Sigma) and the suspension was agitated for 30 minutes followed by aging for 24 hours. It was assumed that the formed $\mathrm{CaP}$ was hydroxyapatite $\left(\mathrm{Ca}_{10}\left(\mathrm{PO}_{4}\right)_{6}(\mathrm{OH})_{2}\right)$ and the formed $\mathrm{CaP}$ was controlled at 16 wt.\% $(\mathrm{CaP} / \mathrm{Silk})$. The silk/nano-CaP scaffold was prepared by addition of $2.0 \mathrm{~g}$ of sodium chloride particles (500-1000 $\mu \mathrm{m}$ ) into $1 \mathrm{~mL}$ of the suspension in a silicon tube ( $9 \mathrm{~mm}$ inner diameter, Deltalab), followed by drying the construct for 2 days. Afterwards, the silk/nano-CaP scaffolds were removed from the silicon tube after immersion in distilled water for 6 hours and, the scaffolds were cut into pieces ( 4 $\mathrm{mm}$ in length). The silk/nano-CaP scaffolds were placed into a new silicon mold. The bilayered scaffolds were formed by adding $300 \mu \mathrm{L}$ aqueous silk solution onto the top of each silk/nano-CaP scaffold, followed by addition of $600 \mathrm{mg}$ sodium chloride particles into the silk solution. The bilayered constructs were dried for 1 day and then extracted in distilled water for 48 hours to remove the salt and by-products. The skin of the scaffolds was removed by a stainless steel punch, and the final scaffolds were formed by freeze-drying (Telstar Cryodos 80, Spain). Single layer silk or silk/nano-CaP scaffolds were also prepared as controls. The bilayered scaffolds were observed by scanning electron microscopy (SEM, NanoSEM-FEI Nova 200, USA) and micro-computed tomography (Micro-CT, Skyscan 1072, Belgium). The hydration degree was analyzed by immersion the scaffolds in $0.9 \%$ sodium chloride solution for 12 hours at $37^{\circ} \mathrm{C}$, and defined as Eq. 1:

Hydration degree $=\left(\mathrm{W}_{\mathrm{w}}-\mathrm{W}_{\mathrm{i}}\right) / \mathrm{Wi}^{*} 100 \%$.

The $\mathrm{W}_{\mathrm{i}}$ and $\mathrm{W}_{\mathrm{w}}$ were the initial dry weight and wet weight of the scaffolds, respectively. The mechanical properties were tested by a universal testing machine (Instron 4505). Bioactivity was performed by immersion the scaffolds in a simulated body fluid solution (SBF) for 14 days [7].

\section{Results and Discussion}
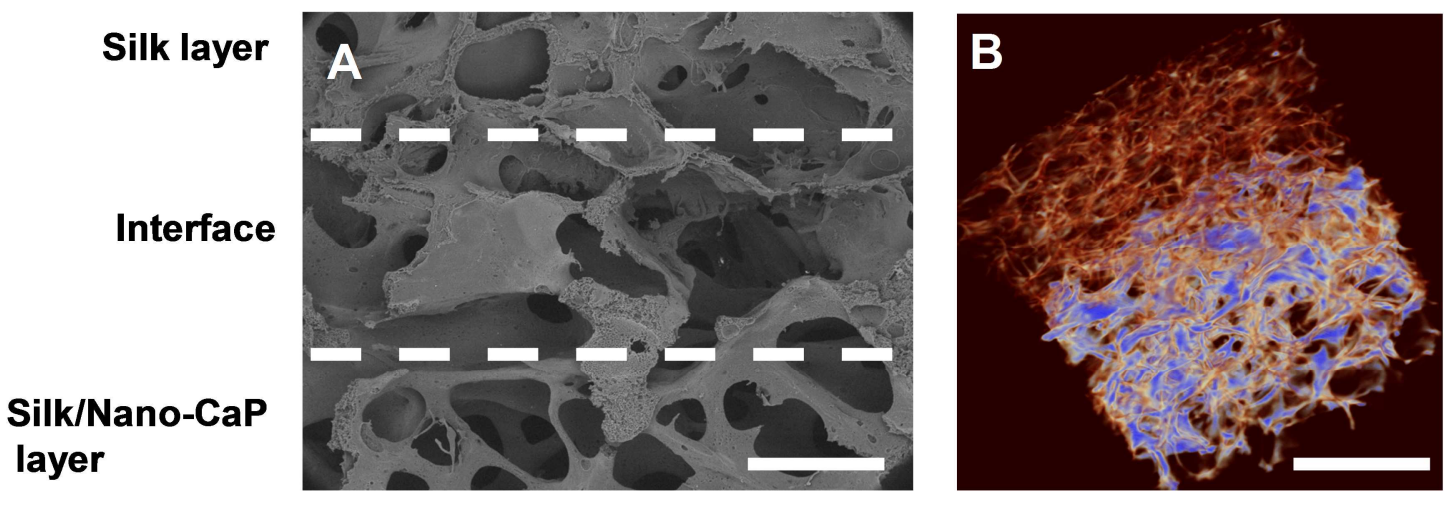

Fig. 1: SEM image (A) and micro-CT image (B) of silk fibroin and silk/nano-CaP bilayered scaffolds. In (B), the blue domain indicates the $\mathrm{CaP}$ phase in the silk/nano-CaP layer and the redbrown domain indicates silk fibroin phase in the bilayered scaffold. Scale bar: $500 \mu \mathrm{m}(\mathrm{A})$ and 3 $\mathrm{mm}(\mathrm{B})$.

In this study, we aimed at producing bilayered silk based scaffolds for OCD regeneration. It was found that the bilayered scaffold presented a continuous porous and interconnected structure in the silk layer, the interface, and the silk/nano-CaP layer (Fig. 1A). The pore size was around $500 \mu \mathrm{m}$. The top layer and the bottom layer integrated very well and without obvious border. The micro-CT images (Fig. 2B) also confirmed the porous structure of the bilayered scaffold. The CaP phase was only observed in the bottom layer (blue) and distributed homogeneously in the silk matrix (red- 
brown). It has been reported in our previous study that the in-situ synthesized CaP particles in that silk/CaP scaffolds presented size around $200 \mathrm{~nm}$ [7]. The in-situ synthesis method using a highly concentrated and viscous silk fibroin aqueous solution allowed to achieve a nano-sized CaP finely dispersed in the silk matrix. The data presented here are illustrative of the good dispersion of formed nano-CaP particles in the scaffold.
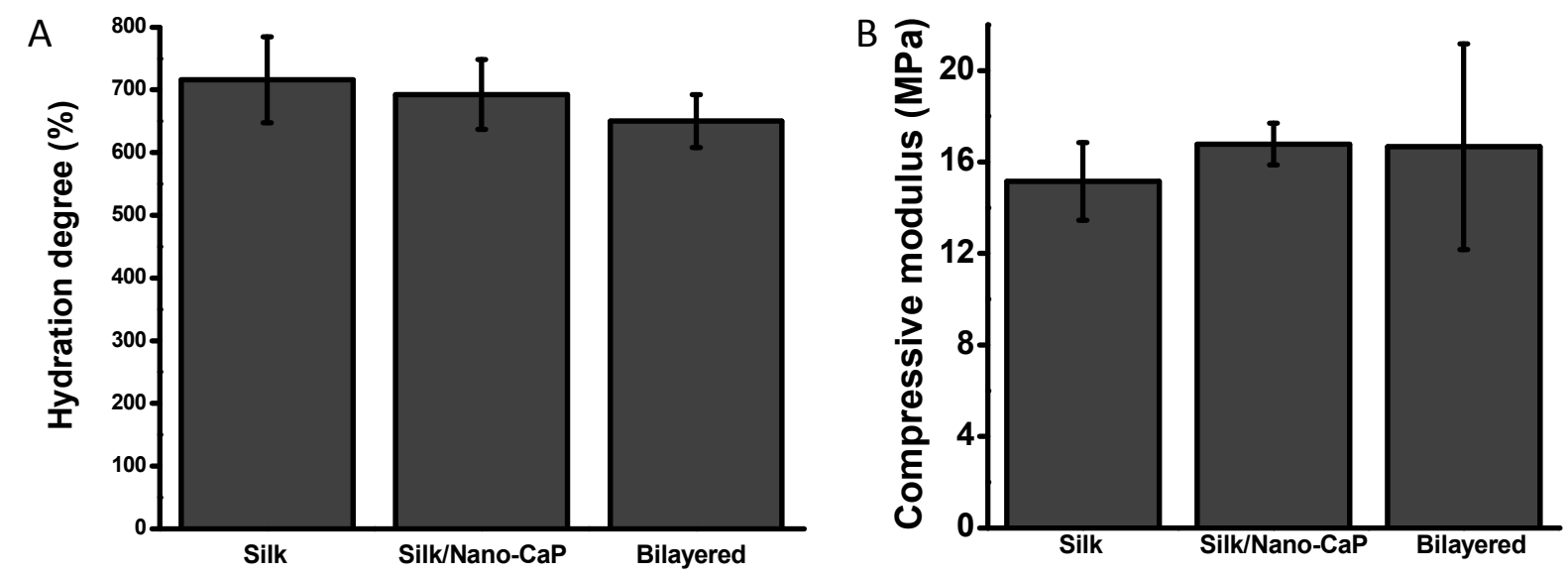

Fig. 2: Hydration degree (A) and compressive modulus (B) of the bilayered scaffolds and single layer scaffolds. Silk: silk scaffolds; Silk/Nano-CaP: silk/nano-CaP scaffolds; Bilayered: bilayered scaffolds.

Water absorbance and wetting properties of the scaffolds play important role for this particular biological application. It was observed that the silk based scaffolds can hydrate in a few hours (data not show) and can reach equilibrium in 12 hours. As showed in Fig. 2A, the bilayered scaffolds can absorb much higher amount of water as compared to its dry weight, and there were no significant differences among the single layer and bilayered scaffolds. The hydration degree is related with two factors, the porous structure of the scaffolds and the wetting properties of the material. Since the bilayered scaffolds were produced by salt leaching approach, they present a high porous and interconnected structure. Furthermore, some hydrophilic groups in the side chains of silk contributed for a higher wetting ability. These are the two factors that contributed to the hydration degree of the scaffolds. On the other hand, the mechanical properties of the scaffolds are essential for tissue regeneration. The rationale to use concentrated silk solution for the preparation scaffolds was to improve the mechanical properties of silk scaffolds. It was found that the compressive modulus of the bilayered scaffolds were comparable to the ones of the single layer scaffolds (Fig. 2B). These results confirm the suitability of the present bilayered scaffolds as implantation materials for OCD.

The combination of calcium phosphates with polymer-based systems is an attractive strategy used for bone tissue engineering to promote an osteoconductivity [5,8]. In order to confirm the osteoconductive potential of bilayered scaffolds, an in vitro bioactivity test was performed. It was showed that the silk/nano-CaP layer can induce the cauliflower-like apatite crystals formation (Fig. 3A), but there was no crystal formed on the silk layer even after 2 weeks (Fig. 3B). These results indicated that the silk/nano-CaP layer may be fast integrated with host subchondral bone tissue and subsequently facilitate the OCD regeneration. The fast integration of the implantation in OCD is critical for cartilage regeneration since it is a big challenge to anchor the implant in the cartilage defect. 

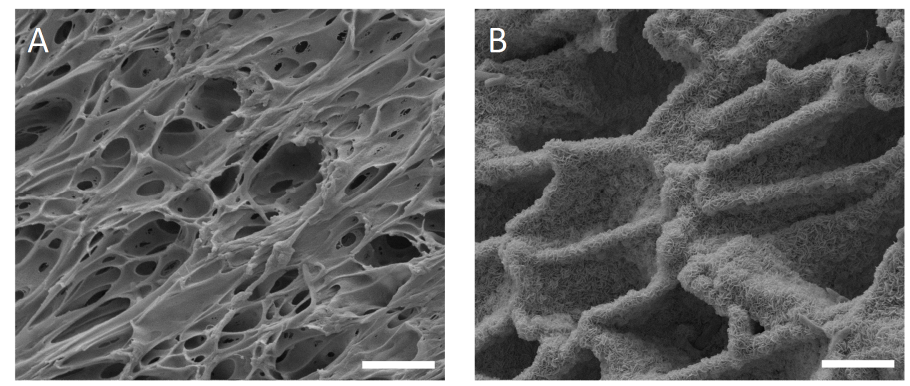

Fig. 3: SEM images of in vitro mineralization of the bilayered scaffolds by immersion in the simulated body fluid solution for 2 weeks. (A): silk layer; (B): silk/nano-CaP layer. Scale bar: $5 \mu \mathrm{m}$.

\section{Conclusions}

In this study, bilayered silk and silk/nano-CaP scaffolds with good integration, proper hydration degree, and superior mechanical properties were successfully developed. These scaffolds show localized osteoconductivity properties in the bone-like layer which is suitable for the proper integration of the scaffolds with the subchondral bone. The bilayered scaffolds can be a promising scaffold candidate for OCD tissue engineering.

\section{Acknowledgements}

This study received funding from Portuguese Foundation for Science and Technology (FCT) through the projects OsteoCart (PTDC/CTM-BPC/115977/2009) and TISSUE2TISSUE (PTDC/CTM/105703/2008). Research leading to these results has received funding from the European Union's Seventh Framework Programme (FP7/2007-2013) under grant agreement $n^{\circ}$ REGPOT-CT2012-316331-POLARIS. Le-Ping Yan is a FCT PhD scholarship holder (SFRH/BD/64717/2009).

\section{References}

[1] F.T. Moutos, L.E. Freed, F. Gullak, A biomimetic three-dimensional woven composite scaffold for functional tissue engineering of cartilage, Nat Mater. 6 (2007) 162-167.

[2] J.F. Mano, R.L. Reis, Osteochondral defects: present situation and tissue engineering approaches, J. Tissue. Eng. Regen. Med. 1 (2007) 261-273.

[3] S. Panseri, A. Russo, C. Cunha, A. Bondi, A.D. Martino, S. Patella, E. Kon, Osteochondral tissue engineering approaches for articular cartilage and subchondral bone regeneration, Knee. Surg. Sport. Tr. A. 20(2012) 1182-1191.

[4] R. Langer, J.P. Vacanti, Tissue engineering, Science. 260(1993) 920-926.

[5] J.M. Oliveira, M.T. Rodrigues, S.S. Silva, P.B. Malafaya, M.E. Gomes, C.A. Viegas, I.R. Dias, J.T. Azevedo, J.F. Mano, R.L. Reis, Novel hydroxyapatite/chitosan bilayered scaffold for osteochondral tissue-engineering applications: Scaffold design and its performance when seeded with goat bone marrow stromal cells, Biomaterials. 27(2006) 6123-6137.

[6] LP. Yan, J.M. Oliveira, A.L. Oliveira, S.G. Caridade, J.F. Mano, R.L. Reis, Macro/microporous silk fibroin scaffolds with potential for articular cartilage and meniscus tissue engineering applications, Acta. Biomater. 8(2012) 289-301.

[7] LP. Yan, J. Silva-Correia, C. Correia, S.G. Caridade, E.M. Fernandes, R.A. Sousa, J.F. Mano, J.M. Oliveira, A.L. Oliveira, R.L. Reis, Bioactive macro/micro porous silk fibroin/nano-sized calcium phosphate scaffolds with potential for bone-tissue-engineering applications, NanomedicineUK. 8(2013) 359-378.

[8] A.L. Oliveira, P.B. Malafaya, S.A. Costa, R.A. Sousa, R.L. Reis, Micro-computed tomography $(\mu-\mathrm{CT})$ as a potential tool to assess the effect of dynamic coating routes on the formation of biomimetic apatite layers on 3D-plotted biodegradable polymeric scaffolds, J. Mater. Sci-Mater. M. 18(2007) 211-223. 